\title{
Comparison of Dopants for Charge Exchange Ionization of Nonpolar Polycyclic Aromatic Hydrocarbons with Reversed-Phase LC-APPI-MS
}

\author{
Derek R. Smith, Damon B. Robb, and Michael W. Blades \\ Department of Chemistry, University of British Columbia, Vancouver, Canada
}

Atmospheric pressure photoionization (APPI) is capable of ionizing nonpolar compounds in LC/MS, through charge exchange reactions following photoionization of a dopant. Recently, several novel dopants-chlorobenzene, bromobenzene, 2,4-difluoroanisole, and 3-(trifluoromethyl)anisole- have been identified as having properties making them wellsuited to serve as dopants for charge exchange ionization under reversed-phase LC conditions. Here, we report the results of experiments comparing their effectiveness to that of established dopants-toluene, anisole, and a toluene/anisole mixture, for the charge exchange ionization of model nonpolar compounds-the 16 polycyclic aromatic hydrocarbons (PAHs) identified by the US EPA as priority pollutants-when using a conventional reversed-phase LC method. Chloro- and bromobenzene were found to be much more effective than toluene for all the PAHs, due to the relatively low reactivity of their photoions with the solvent. Their overall performance was also better than that of anisole, due to anisole's ineffectiveness toward higher-IE compounds. Further, the experiments revealed that anisole's performance for higher-IE compounds can be dramatically improved by introducing it as a dilute solution in toluene, rather than neat. The two fluoroanisoles provided the highest overall sensitivity, by a slim margin, when introduced as dilute solutions in either chloro- or bromobenzene. (J Am Soc Mass Spectrom 2009, 20, 73-79) (c) 2009 Published by Elsevier Inc. on behalf of American Society for Mass Spectrometry

A tmospheric pressure photoionization (APPI) is an ionization method for LC/MS suitable for both polar and nonpolar compounds [1-3]. With APPI, analyte ionization is mostly due to ionmolecule reactions following photoionization of a primary reagent, typically a dopant [4]. Analyte ionization can occur in positive mode through either proton transfer or charge exchange (electron-transfer) reaction pathways. This article regards the ionization of nonpolar, low proton affinity compounds via charge exchange with dopant radical cations.

In APPI, for charge exchange ionization to occur the dopant's ionization energy (IE) must be greater than that of the analyte and to be efficient its radical cations must not be consumed through reactions with the solvent, its own neutrals, or impurities. Toluene was the first dopant to be used for charge exchange ionization in APPI [1], and it has a relatively high IE $(8.83 \mathrm{eV})$, making it suitable for a wide range of analytes (all IE values are from reference [5]). In practice, however, toluene is only an efficient charge exchange dopant under normal-phase $[6,7]$ and/or low-flow conditions

Address reprint requests to Dr. M. W. Blades, Department of Chemistry, University of British Columbia, 2036 Main Mall, Vancouver, B.C., Canada V6T 1Z1. E-mail: blades@chem.ubc.ca
[8] because its radical cations are rapidly consumed in reactions with methanol and acetonitrile at conventional LC flow rates $[6,9,10]$. Some other dopant is then required for efficient charge exchange ionization with reversed-phase LC methods. The usual alternative to toluene for promoting charge exchange ionization is anisole, whose photoions are stable in the presence of methanol and acetonitrile [11]. Anisole, however, has a relatively low IE $(8.20 \mathrm{eV})$, restricting its applicability. Mixtures of anisole and toluene have been utilized by Itoh et al. as dopants to promote charge exchange ionization under reversed-phase conditions, in the analysis of the 16 polycyclic aromatic hydrocarbons (PAHs) identified by the US EPA as priority pollutants [12]. In this case, multicomponent dopants were examined after initial experiments established that, even if imperfect, toluene was still the best dopant for the early-eluting, high-IE PAHs naphthalene $(8.14 \mathrm{eV})$ and acenaphthylene $(8.12 \mathrm{eV})$, while anisole was best for the others. Itoh et al. envisioned that by mixing toluene and anisole, the strengths of one could compensate for the weaknesses of the other; in the end, an anisole/toluene mixture of 0.5:99.5 (vol/vol) was empirically determined to give the best overall ionization of the PAHs. To our knowledge, besides from toluene, anisole, and mixtures of the two, there have been no other dopants utilized to date
(C) 2009 Published by Elsevier Inc. on behalf of American Society for Mass Spectrometry. $1044-0305 / 09 / \$ 32.00$

doi:10.1016/j.jasms.2008.09.012
Published online September 19, 2008 Received August 15, 2008 Revised September 10, 2008 Accepted September 11, 2008 
for charge exchange ionization in applications involving reversed-phase LC methods.

As a result of recent efforts to extend the utility of APPI in the analysis of nonpolar compounds, several novel dopants for promoting charge exchange ionization with reversed-phase LC methods have been identified. In a first study, both chlorobenzene (IE $=9.07 \mathrm{eV})$ and bromobenzene $(\mathrm{IE}=9.00 \mathrm{eV}$ ) were shown to yield photoions at least partially stable in the presence of methanol and acetonitrile [13]. Subsequently, a number of fluoro-substituted anisoles, including 2,4-difluoroanisole (DFA) and 3-(trifluoromethyl)anisole (TFMA), were also shown to yield photoions stable in the reversedphase solvents [14], much like anisole itself; DFA and TFMA, however, have IEs elevated above anisole's by their electron-withdrawing (EW) fluorines (their exact IEs are unknown, but they are presumed to be above anisole's because a single fluorine gives 3-fluoroanisole an IE of $8.4 \mathrm{eV}$ and the effects of EW substituents are generally additive - see reference [14] and references therein). The photoions of both DFA and TFMA are less reactive with methanol than those of chloro- and bromobenzene, suggesting that these may be the more efficient charge exchange dopants under some conditions. On the other hand, unlike chloro- and bromobenzene, neither DFA nor TFMA is suitable for use in neat form, because when used at ordinary dopant flow rates their photoions are consumed in reactions presumed to be with accompanying impurities, at least in the batches used to date [14]. This does not, however, preclude their being used to advantage as components in a dopant mixture, as we shall demonstrate below. Thus, chloroand bromobenzene, and-impurities notwithstandingDFA and TFMA, all satisfy important requirements for use as charge exchange dopants under reversed-phase conditions: they have relatively high IEs and yield radical cations stable in the presence of methanol and acetonitrile. Here, we provide the first report on the effectiveness of these novel dopants relative to that of the established dopants for the charge exchange ionization of model nonpolar compounds, PAHs, using a conventional reversed-phase LC method.

\section{Experimental}

\section{Chemicals}

The dopants tested were 2,4-difluoroanisole (DFA; 99\%) from Acros Organics (Morris Plains, NJ), bromobenzene $(\geq 99.5 \%)$ and anisole $(\geq 99 \%)$ from Fluka (Buchs, Switzerland), chlorobenzene (99.9\%) and 3-(trifluoromethyl)anisole (TFMA; 99\%) from Sigma-Aldrich (St. Louis, MO), and HPLC-grade toluene $(99.9 \%)$ from Fisher Scientific (Fair Lawn, NJ). DFA and TFMA were introduced to the ion source as dilute solutions, $0.5 \%$ ( $\mathrm{vol} / \mathrm{vol})$, in each of bromo- and chlorobenzene. The solvents used for the sample solutions and chromatography were HPLC-grade acetonitrile, methanol, and hexanes from Fisher Scientific, and deionized water from an in-house generator. For the main LC/MS experiments, the sample solution was an EPA 610 polynuclear aromatic hydrocarbons mix (catalog no. 4S8743) from Supelco (Bellefonte, PA), diluted $1000 \times$ in methanol/water (60:40, vol/vol); the 16 PAHs in the mix are listed in alphabetical order in Table 1, with their molecular weights and final concentrations. For the supplementary infusion experiments, individual stock solutions of pyrene, benzo[a]pyrene, naphthalene, acenaphthylene, fluorene, phenanthrene, and fluoranthene were prepared in hexanes. The concentrations of the stock solutions were all $500 \mathrm{ng} \mu \mathrm{L}^{-1}$, except for naphthalene's, which was $5000 \mathrm{ng} \mu \mathrm{L}^{-1}$. Individual sample solutions were obtained for all the compounds by diluting their stock solutions $100 \times$ with methanol/ water (90:10); additional sample solutions were made for pyrene and benzo[a]pyrene by diluting their stock solutions $100 \times$ with acetonitrile. The final concentrations of all the sample solutions were then $5 \mathrm{ng} \mu \mathrm{L}^{-1}$, except for naphthalene's, which was $50 \mathrm{ng} \mu \mathrm{L}^{-1}$ (because of its weaker response with the dopants utilized in the infusion experiments). The individual PAHs were from Sigma-Aldrich, except for pyrene, which was from Fluka. Aside from the various dilutions, all of the chemicals were used as received.

\section{Methods}

The main LC/MS experiments consisted of acquiring chromatograms of the PAH mixture using each of the dopants. The aim of the experiments was to compare the sensitivities attainable for each PAH in the mix using each dopant. Accordingly, the basis for comparison was absolute signal intensity, preventing the use of internal standards and necessitating the monitoring of intra- and interday drift in instrument response. To this end, in the final experiments the dopants were divided into three groups: (1) toluene, anisole, and anisole/

Table 1. The PAHs of EPA 610

\begin{tabular}{lcc}
\hline \multicolumn{1}{c}{ Compound } & MW $(\mathrm{u})$ & Conc. $\left(\mathrm{ng} \mu \mathrm{L}^{-1}\right)^{\mathrm{b}}$ \\
\hline \hline Acenaphthene & 154 & 1.0 \\
Acenaphthylene & 152 & 2.0 \\
Anthracene & 178 & 0.10 \\
Benzo[a]anthracene & 228 & 0.10 \\
Benzo[a]pyrene & 252 & 0.10 \\
Benzo[ $b]$ fluoranthene & 252 & 0.20 \\
Benzo[g,h,i]perylene & 276 & 0.20 \\
Benzo[k]fluoranthene & 252 & 0.10 \\
Chrysene & 228 & 0.10 \\
Dibenzo[a,h]anthracene & 278 & 0.20 \\
Fluoranthene & 202 & 0.20 \\
Fluorene & 166 & 0.20 \\
Indeno[1,2,3-cd]pyrene & 276 & 0.10 \\
Naphthalene & 128 & 1.0 \\
Phenanthrene & 178 & 0.10 \\
Pyrene & 202 & 0.10 \\
\hline
\end{tabular}

aThe compounds are listed in alphabetical order.

${ }^{b}$ Final concentration in injected sample volume. 
Table 2. LC method

\begin{tabular}{cccrr}
\hline Step & $\begin{array}{c}\text { Total time } \\
(\text { min })\end{array}$ & $\begin{array}{c}\text { Flow rate } \\
\left(\mu \mathrm{L} \mathrm{min}^{-1}\right)\end{array}$ & A (\%) & B (\%) \\
\hline \hline 0 & $18^{\mathrm{a}}$ & 200 & 100.0 & 0.0 \\
1 & 1.0 & 200 & 100.0 & 0.0 \\
2 & 7.0 & 200 & 60.0 & 40.0 \\
3 & 11.0 & 200 & 0.0 & 100.0 \\
4 & 42.0 & 200 & 0.0 & 100.0 \\
5 & 44.0 & 200 & 100.0 & 0.0 \\
\hline
\end{tabular}

${ }^{a}$ Column equilibration period (preinjection).

toluene; (2) bromobenzene, TFMA/bromobenzene, and DFA/bromobenzene; and (3) chlorobenzene, TFMA/ chlorobenzene, and DFA/chlorobenzene (the mixed dopants were all 0.5:99.5, vol/vol). One group of dopants was tested per day. To monitor intraday drift, on the day a particular group was being tested, one chromatogram was obtained using each of the dopants in the group, and then the series was repeated twice; in this way, three replicates for each dopant were attained at different times throughout the day. Toluene was an exceptional case in that its replicates were acquired consecutively at the start of its day, on which anisole was also used, due to the long time required for anisole residue in the system to dissipate and the great effect that anisole has on response. To monitor interday drift, a day of control experiments was also performed for a group composed of one dopant from each of the original groups-specifically, anisole/toluene, bromobenzene, and chlorobenzene; the extent of interday drift was assessed by comparing the results of the day of control experiments with those of the days when each control dopant was tested with its own group. Lastly, to examine more closely the effect of the anisole/toluene mixing ratio on the response of select PAHs, supplementary experiments involving the infusion of the individual PAH solutions were performed.

\section{Instrumentation}

The mass spectrometer was an API 3200 triplequadrupole from Applied Biosystems/MDS SCIEX (Concord, Ontario, Canada), equipped with a PhotoSpray APPI source. The APPI source's transfer voltage was $700 \mathrm{~V}$, its probe temperature was $400{ }^{\circ} \mathrm{C}$, and its nebulizer and lamp gases were set to 60 and 20 psi, respectively. The orifice (declustering) potential was set to a low value, $10 \mathrm{~V}$, to minimize collision-induced dissociation. For the LC/MS experiments, the scan type was "Q1 Multiple Ions," the polarity was positive, and the Q1 resolution was "unit." The masses scanned were $128,152,154,166,178,202,228,252,276$, and $278 \mathrm{u}$, corresponding to the $\mathrm{M}^{+\cdot}$ ions of the various PAHs. The dwell time for each mass was $100 \mathrm{~ms}$. For the infusion experiments, where analytes were introduced individually, all scan parameters were the same, except that the dwell time at the mass of the $\mathrm{M}^{+\cdot}$ ion was $1000 \mathrm{~ms}$ and 30 scans were acquired.
For the LC experiments, the solvent pump was a model 1100 G1311A from Agilent (Palo Alto, CA), and the column was an Inertsil ODS-P $250 \mathrm{~mm} \times 2.1 \mathrm{~mm}, 5$ $\mu \mathrm{m}$ particle size, from GL Sciences, Inc. (Tokyo, Japan). The column was at room temperature. Solvent A was methanol/water (90:10) and Solvent B was acetonitrile. The LC method used a constant flow rate of $200 \mu \mathrm{L}$ $\min ^{-1}$ and a two-step gradient, summarized in Table 2 (an extra-long column equilibration period of $18 \mathrm{~min}$ was included to allow for removal of the dopant from the prior run, via flushing and baking). Sample injections were made using the valve integrated into the mass spectrometer; the injection volume was $10 \mu \mathrm{L}$. For the supplementary infusion experiments, $10 \mu \mathrm{L} \mathrm{min}^{-1}$ analyte solution and $190 \mu \mathrm{L}$ min $^{-1}$ make-up solvent (either methanol/water, 90:10, or acetonitrile) were delivered separately via syringe pumps from Harvard Apparatus (Holliston, MA), and combined with a tee before mixing with the dopant. For all the experiments, dopant was delivered at $20 \mu \mathrm{L} \min ^{-1}$ using the mass spectrometer's integrated syringe pump. Dopant was combined with the solvent before entering the ion source using a tee, post-column in the case of the LC experiments, to circumvent a suspected carry-over problem with the source's dopant introduction port, which was plugged during the experiments.

\section{Results and Discussion}

\section{Dopant Comparisons}

Figure 1 is a representative chromatogram of the PAH mixture, showing a peak for each of the 16 PAHs. The peaks are all well-resolved, indicating that the chromatographic method was sufficient to prevent interferences as a result of coeluting compounds. The dopant in this case was DFA/bromobenzene (0.5:99.5), which was

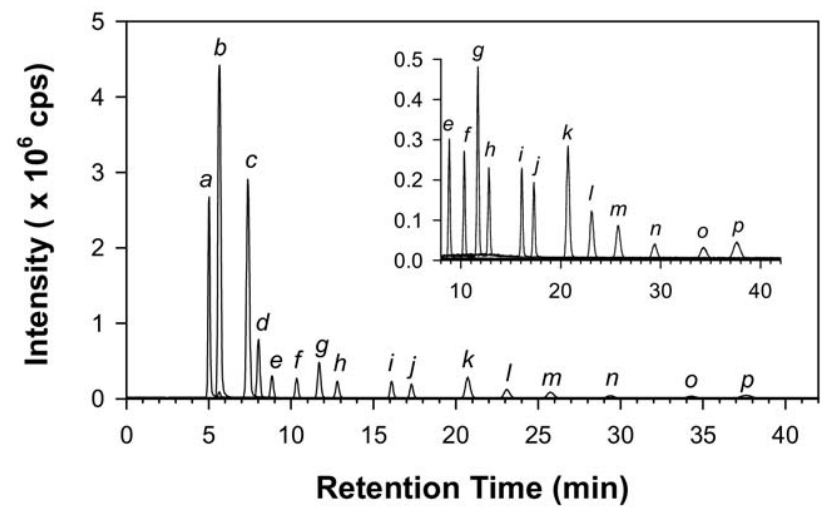

Figure 1. Chromatogram of the 16 PAHs obtained using 2,4difluoroanisole (DFA)/bromobenzene (0.5:99.5, vol/vol) as the dopant. The peaks are due to (a) naphthalene, (b) acenaphthylene, (c) acenaphthylene, (d) fluorene, (e) phenanthrene, (f) anthracene, (g) fluoranthene, (h) pyrene, (i) benzo[ $a]$ anthracene, (j) chrysene, (k) benzo[b]fluoranthene, (1) benzo[k]fluoranthene, (m) benzo$[a]$ pyrene, (n) dibenzo[a,h]anthracene, (o) indeno[1,2,3-cd]pyrene, and (p) benzo[ghi]perylene. 
found to provide the highest overall sensitivity. Differences in peak heights for the PAHs, particularly between naphthalene, acenaphthylene, and acenaphthylene (peaks $a-c$ ) and the others, are largely attributable to differences in their concentrations (see Table 1). Significantly, the peaks for the high-IE PAHs naphthalene and acenaphthylene are very large, a result attributable in part to their high concentrations, but also to the fact that they are efficiently ionized via DFA/ bromobenzene. To our knowledge, this is the first published demonstration of efficient charge exchange ionization of such high-IE compounds using APPI with a reversed-phase LC method.

Table 3 presents a summary of the dopant comparison results. For each PAH, the table includes its IE and the chromatographic peak areas obtained with the various dopants, normalized to the value obtained with the dopant providing the best sensitivity for it. The PAHs are listed in order of decreasing IE. The dopants are organized according to the group with which they were tested, on a given day, as described above. Regarding uncertainty, generally, the intraday relative standard deviations of the peak area determinations were $2 \%$ or less, whereas the control experiments revealed that the interday drift was larger, up to about $9 \%$; thus, quantitative comparisons between dopants of the same group are more certain than those between dopants of different groups.

The results for toluene indicate that it was able to ionize all the PAHs, but with very low efficiency relative to the best of the other dopants. As is wellestablished, toluene photoions are effective charge exchange reagents for a wide range of compounds, but they are lost in reactions with methanol and/or acetonitrile, lowering the rate of analyte ionization and thus ionization efficiency. Anisole, on the other hand, was nearly or actually the best dopant for 10 of the PAHs, but only for those with IEs $\leq 7.6 \mathrm{eV}$ (the IEs of the bottom three PAHs are presumed to be low because of their large size and the trend towards decreasing IE with increased size in aromatic compounds [15]). The results for the other six PAHs indicate that anisole became much less effective as the IE of the compound increased, and the magnitude of $\Delta \mathrm{IE}$ for the reaction decreased, to the extent that it was entirely ineffective for naphthalene. Indeed, prior studies have shown that as the magnitude of $\Delta \mathrm{IE}$ decreases, the overall rate of an exothermic charge exchange reaction may be reduced, either because the rate constant of the forward reaction is lowered below the collision frequency or else because the rate of the reverse endothermic reaction becomes significant $[16,17]$. Note that the latter phenomenon is likely to be exacerbated by the large amount of neutral dopant generally present in the source, which may be expected to drive the equilibrium towards the reactants. With the anisole/toluene mix, however, the sensitivities for all the higher-IE PAHs except naphthalene were greatly increased, relative to those attainable with either neat toluene or neat anisole. Even naphthalene was

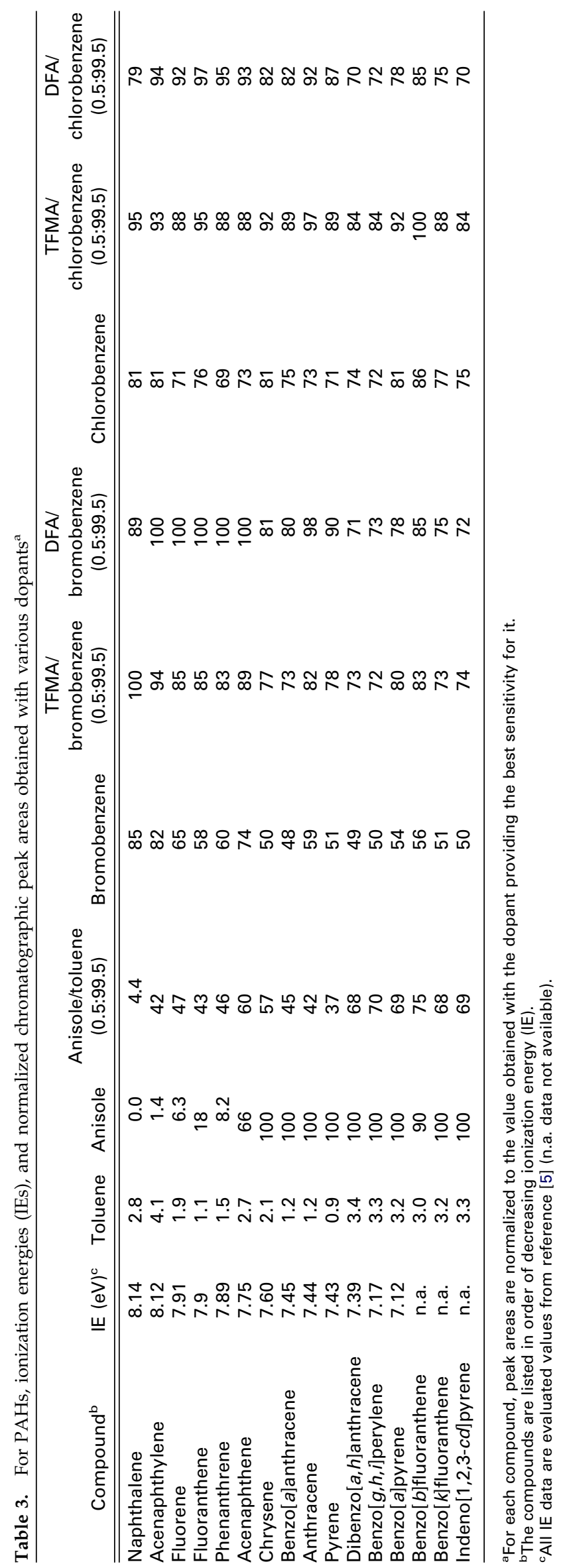


better ionized with the $0.5 \%$ anisole than with neat toluene, though the improvement was much smaller. The results for the anisole/toluene mix then prove that small $\Delta \mathrm{IE}$ alone are not generally responsible for neat anisole's poor performance for high-IE PAHs, since anisole is clearly able to ionize all of them, at least to an extent, under some conditions. Thus, one or more additional factors must also affect the results in the neat anisole case. As for the low-IE PAHs best ionized by neat anisole, dilution of the anisole with toluene had a slight adverse effect on their ionization. Altogether, the results for toluene, anisole, and the anisole/toluene mix indicate that the concentration of anisole in the dopant may have a strong effect on analyte ionization efficiency, depending upon the IE of the compound, while compounds having an IE closely approaching that of anisole, such as naphthalene, may not be efficiently ionizable using any of them.

Bromobenzene provided an obvious advantage over the best of the established dopants in that it provided enhanced sensitivity for compounds with high-IE, about $20 \times$ higher for naphthalene, and also fairly high sensitivity for those with lower IEs. The improved performance for naphthalene in particular is likely attributable to the much higher IE of bromobenzene relative to anisole, coupled with how $\Delta \mathrm{IE}$ may affect reaction rates, described above. This performance also hinges upon the appreciable stability of the bromobenzene photoions under reversed-phase conditions. The results for the fluoroanisole/bromobenzene mixtures show that further improvement is possible: for every $\mathrm{PAH}$, a slight but significant increase in sensitivity was obtained by adding $0.5 \%$ of either TFMA or DFA to bromobenzene. The increases in sensitivity are attributable to the fact that the radical cations of the fluoroanisoles are less reactive with the solvent than those of bromobenzene and are thus more likely to survive in the source to serve as reagents for charge exchange ionization. As for the chlorobenzene results, these were very similar to those of bromobenzene, except that chlorobenzene provided slightly higher sensitivity for the mid- and low-IE PAHs. For chlorobenzene, the beneficial effect of adding TFMA and DFA was still evident for many of the higher-IE PAHs but the ionization of the lower-IE PAHs was little affected. Of the new dopants, the DFA/bromobenzene mix appeared to provide the highest overall sensitivity, being the best of the group for the high-IE PAHs and very good for the others.

The fluoroanisoles were introduced as dilute solutions in chloro- and bromobenzene in an effort to maximize the production of their radical cations while minimizing the introduction of interferent impurities (recall that when the fluoroanisoles are used neat, their photoions are consumed in reactions presumed to be with accompanying impurities). The mechanism for this mixed-dopant approach involves a rapid cascade of charge from primary photoions of the bulk dopant to neutrals of the trace dopant having a lower IE, thereby generating an abundance of dopant radical cations from the impure dopant without having to introduce an abundance of impurities. The data for the fluoroanisole/halobenzene mixtures prove that this approach may be used to increase sensitivity. Thus, in the event that an impurity in a desired dopant interferes with analyte ionization, it may be advantageous to dilute that dopant in another having a higher IE and lacking interfering impurities.

\section{Effect of Percent Anisole}

We now take a closer look at the effect of the percentage of anisole in anisole/toluene dopant mixtures, to investigate further the result indicating that in some cases $0.5 \%$ anisole may be better than either neat anisole or toluene. Figure 2 illustrates the effect of $\%$ anisole on the normalized peak areas for the PAHs, ordered by decreasing IE, using the data available from the LC/MS

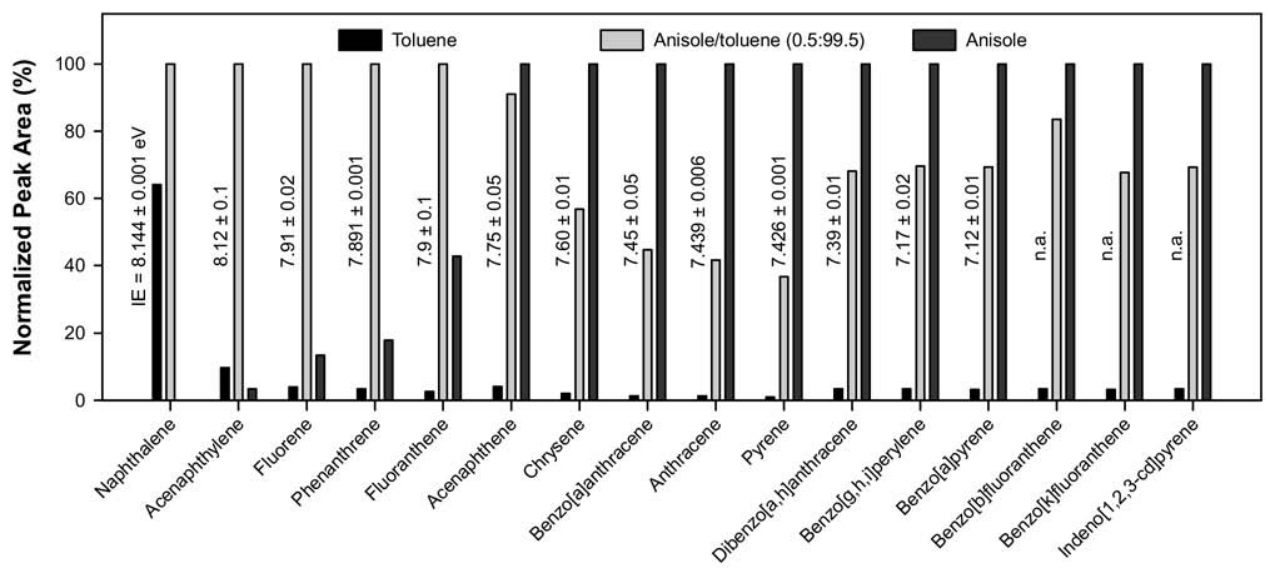

Figure 2. Normalized chromatographic peak areas for the 16 PAHs obtained using each of toluene, anisole/toluene (0.5:99.5), and anisole as the dopant. Peak areas are normalized to the highest value obtained with any of the three dopants. The PAHs are ordered based on decreasing IE (the IE values and the associated uncertainties are the estimated values from reference [5]). 
experiments. For the first 10 PAHs, there is a clear trend in the data: with decreasing IE, there was a monotonic increase in the effectiveness of neat anisole relative to $0.5 \%$ anisole. Note that the relatively large uncertainty in fluoranthene's IE $(7.9 \pm 0.1 \mathrm{eV})$ means there is some latitude in its position relative to the other PAHs; it has been positioned after phenanthrene $(\mathrm{IE}=7.891 \mathrm{eV})$ because of how well this makes its data fit with the prevailing trend in the remainder of the data (the closeness of this fit, taken together with fluoranthene's evaluated IE and the uncertainty in it, suggest that fluoranthene's actual IE is somewhere between 7.89 and $7.80 \mathrm{eV})$. As for the abrupt change for the PAHs following pyrene, for which $0.5 \%$ anisole was relatively more effective than would be anticipated based upon the trend of the first ten PAHs, this appears to be an artifact of the changing solvent composition during the LC run (see the note in the next paragraph).

Figure 3 presents the results of separate infusion experiments where the intensities of select PAHs were monitored individually as a function of the percentage of anisole in the dopant. Solvent A of the LC experiments (methanol/water, 90:10) was used to deliver the PAHs. Figure $3 \mathrm{a}$ shows the results for pyrene and benzo[a]pyrene, chosen to represent the low-IE PAHs: for both compounds, signal intensity increased steadily as $\%$ anisole increased until a plateau was reached at about $5 \%$ anisole, without an optimum. (Note that the effectiveness of $0.5 \%$ anisole relative to $100 \%$ anisole was about the same for both pyrene and benzo[a]pyrene, unlike in the LC experiments, as described in the last paragraph; a control experiment using Solvent B, acetonitrile, instead of Solvent A, showed that the effectiveness of $0.5 \%$ anisole relative to $100 \%$ anisole is higher with acetonitrile than with methanol/water, for both PAHs, indicating that it was the solvent gradient that was mostly responsible for the change following pyrene in Figure 2.) Figure $3 \mathrm{~b}$ shows the results for the five PAHs that were better ionized with $0.5 \%$ anisole than with neat anisole: these all had an optimum \% anisole, beyond which sensitivities decreased steadily with increasing anisole content. Figure $3 c$ presents the same results more clearly, using a log scale on the $\mathrm{x}$-axis. This plot reveals that for maximum sensitivity, the higher the IE of the PAH, the less anisole could be present in the dopant mixture. The optimum \% anisole values span a wide range of concentrations, differing by a factor of 50 , from $0.1 \%$ for naphthalene to $5 \%$ for fluoranthene. This is an important new result, revealing an additional factor to be considered when optimizing methods using anisole as a dopant.

Though elucidating mechanisms was not a primary aim of this study, we can speculate as to the origins of the last result. A first possibility is that there may be one or more impurities in anisole that may react by charge exchange with radical cations of the higher-IE PAHs, neutralizing them, but not those of the PAHs with lower IEs. In full scan spectra acquired under the test conditions, the only peak observed to behave as would
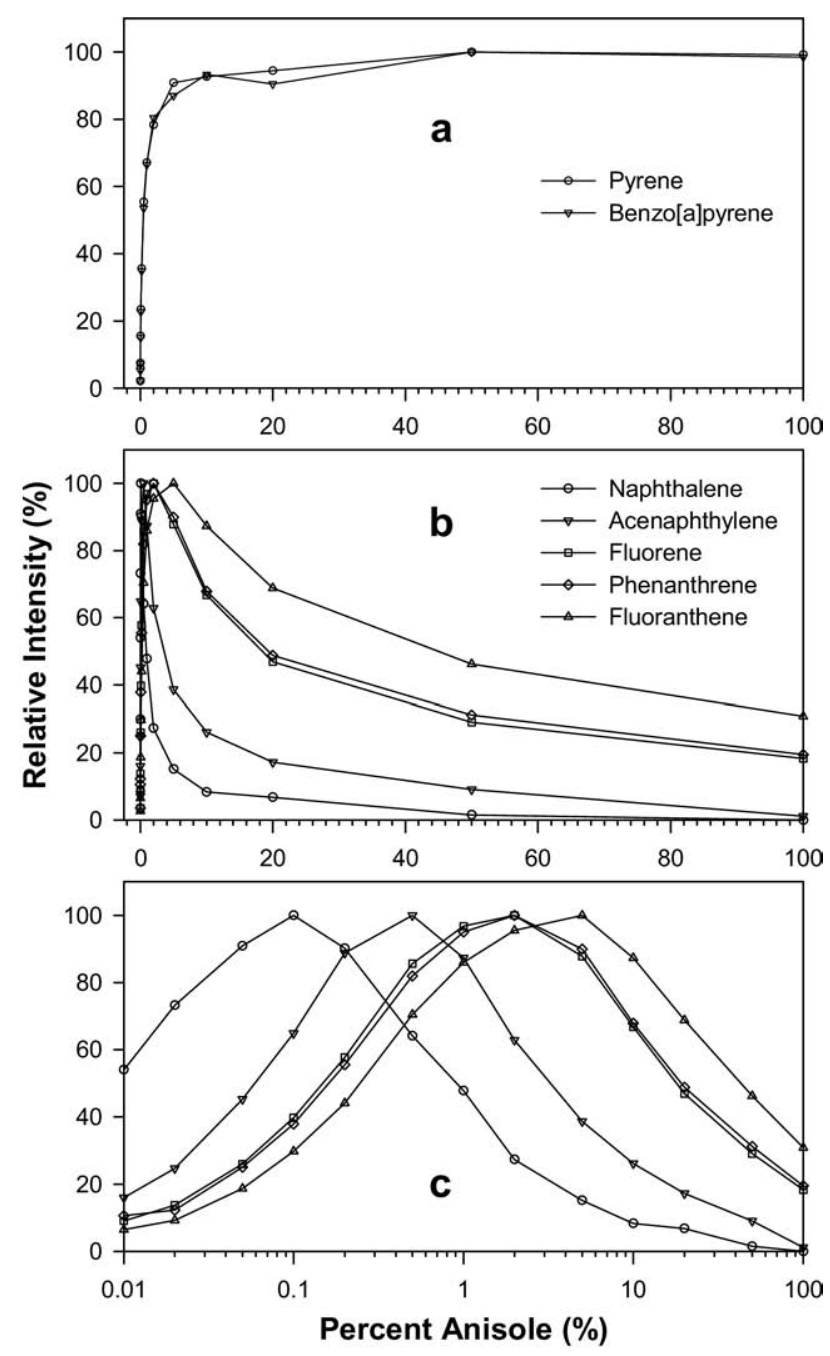

Figure 3. For select PAHs, relative peak intensity versus the percentage of anisole in an anisole/toluene dopant mixture. (a) Pyrene and benzo[a]pyrene are representative of low-IE compounds, having no optimum in peak intensity versus $\%$ anisole. (b) Naphthalene, acenaphthylene, fluorene, phenanthrene, and fluoranthene are relatively high IE compounds, having an optimum \% anisole. (c) The same as (b), but plotted on a log scale for clarity.

be expected for an impurity in anisole, i.e., to increase in intensity relative to anisole's at $m / z 108$ as the \% anisole increased, was at $m / z 122$, possibly due to anisole with a methyl substituent on its ring. Indeed, both 1-methoxy4-methyl benzene and 1-methoxy-2-methyl benzene have evaluated IEs of $7.9 \mathrm{eV}$, which is about the value separating the compounds requiring dilute anisole from those best ionized by neat anisole. Radical cations of the five highest-IE PAHs would certainly be expected to react with these compounds to an extent, at least, while those of the PAHs with substantially lower IEs would not. Hence, there is reason to believe that the negative effect on ionization observed for high-IE PAHs at high $\%$ anisole levels may be due at least in part to methylsubstituted impurities introduced with the anisole. Alternatively, it may be that increasing amounts of neutral 
anisole are detrimental to the ionization of the higher-IE PAHs because of how this may promote the reverse endothermic reaction to reform analyte neutrals, a process expected to be increasingly important as the magnitude of $\triangle \mathrm{IE}$ for the forward reaction (and thus the equilibrium constant) diminishes. Presently, we are unable to say which, if any, of these proposed mechanisms are mostly responsible for the results, and further study will be required to elucidate the actual operative mechanism(s).

\section{Conclusions}

Bromobenzene and chlorobenzene are novel high-IE dopants that can provide efficient charge exchange ionization of nonpolar compounds under reversed-phase LC conditions. 2,4-Difluoroanisole and 3-(trifluoromethyl) anisole may provide even greater sensitivity, when diluted in bromo- or chlorobenzene, though the performance gain is slight and may not be significant in practice. Anisole is a very effective charge exchange dopant for low-IE compounds, but it becomes decreasingly effective for higher-IE analytes, possibly because of one or more impurities that scavenge charge and/or because the reverse endothermic reaction becomes increasingly significant.

\section{Acknowledgments}

The authors acknowledge funding from the Natural Sciences and Engineering Research Council of Canada (NSERC) and the University of British Columbia. They thank Jean-François Alary of MDS-SCIEX for the kind donation of the PhotoSpray source.

\section{References}

1. Robb, D. B.; Covey, T. R.; Bruins, A. P. Atmospheric Pressure Photoionization: An Ionization Method for Liquid Chromatography-Mass Spectrometry. Anal. Chem. 2000, 72, 3653-3659.
2. Syage, J. A.; Evans, M. D.; Hanold, K. A. Photoionization Mass Spectrometry. Am. Lab. 2000, 32, 24-29.

3. Cai, Y.; Kingery, D.; McConnell, O.; Bach II, A. C. Advantages of Atmospheric Pressure Photoionization Mass Spectrometry in Support of Drug Discovery. Rapid Commun. Mass Spectrom. 2005, 19, 1717-1724.

4. Robb, D. B.; Blades, M. W. State-of-the-Art in Atmospheric Pressure Photoionization for LC/MS. Anal. Chim. Acta 2008, 627, 34-49.

5. http://webbook.nist.gov/chemistry.

6. Kauppila, T. J.; Kuuranne, T.; Meurer, E. C.; Eberlin, M. N.; Kotiaho, T.; Kostiainen, R. Atmospheric Pressure Photoionization Mass Spectrometry. Ionization Mechanism and the Effect of Solvent on the Ionization of Naphthalenes. Anal. Chem. 2002, 74, 5470-5479.

7. Müller, A.; Mickel, M.; Geyer, R.; Ringseis, R.; Eder, K.; Steinhart, H. Identification of Conjugated Linoleic Acid Elongation and $\beta$-Oxidation Products by Coupled Silver-Ion HPLC APPI-MS. J. Chromatogr. B 2006, 837, 147-152.

8. Robb, D.; Blades, M. Atmospheric Pressure Photoionization for Ionization of Both Polar and Nonpolar Compounds in Reversed-Phase LC/ MS. Anal. Chem. 2006, 78, 8162-8164.

9. Koster, G.; Bruins, A. P. Mechanisms for ion formation in LC/MS by Atmospheric Pressure Photo-Ionization (APPI). Proceedings of the 49th ASMS Conference on Mass Spectrometry and Allied Topics; Chicago, IL, May, 2001.

10. Robb, D.; Blades, M. Effects of Solvent Flow, Dopant Flow, and Lamp Current on Dopant-Assisted Atmospheric Pressure Photoionization (DA-APPI) for LC-MS. Ionization via Proton Transfer. J. Am. Soc. Mass Spectrom. 2005, 16, 1275-1290.

11. Kauppila, T. J.; Kostiainen, R.; Bruins, A. P. Anisole, a New Dopant for Atmospheric Pressure Photoionization Mass Spectrometry of Low Proton Affinity, Low Ionization Energy Compounds. Rapid Commun. Mass Spectrom. 2004, 18, 808-815.

12. Itoh, N.; Aoyagi, Y.; Yarita, T. Optimization of the Dopant for the Trace Determination of Polycyclic Aromatic Hydrocarbons by Liquid Chromatography/Dopant-Assisted Atmospheric-Pressure Photoionization/ Mass Spectrometry. J. Chromatogr. A 2006, 1131, 285-288.

13. van Dam, A.; Bruins, A. P. New Dopants for Atmospheric Pressure Photoionization under Reversed Phase Liquid Chromatography Conditions. Poster presented at the 21st Montreaux Symposium; November 2004.

14. Robb, D. B.; Smith, D. R.; Blades, M. W. Investigation of SubstitutedBenzene Dopants for Charge Exchange Ionization of Nonpolar Compounds by Atmospheric Pressure Photoionization. J. Am. Soc. Mass Spectrom. 2008, 19, 955-963.

15. Meot-Ner (Mautner), M. Ion Thermochemistry of Low-Volatility Compounds in the Gas Phase. 3. Polycyclic Aromatics: Ionization Energies, Proton and Hydrogen Affinities. Extrapolations to Graphite. J. Phys. Chem. 1980, 84, 2716-2723.

16. Lias, S. G.; Ausloos, P.; Horvath, Z. Charge Transfer Reactions in Alkane and Cycloalkane Systems. Estimated Ionization Potentials. Int. J. Chem. Kinetics 1976, 8, 725-739.

17. Sieck, L. W.; Mautner (Meot-Ner), M. Ionization Energies and Entropies of Cycloalkanes. Kinetics of Free Energy Controlled Charge-Transfer Reactions. J. Phys. Chem. 1982, 86, 3646-3650. 